\title{
Measurements of inclusive neutral diboson production with ATLAS
}

\author{
Stefan Richter*, on behalf of the ATLAS Collaboration \\ DESY, Germany \\ E-mail: stefan.richterecern.ch
}

\begin{abstract}
We review recent ATLAS measurements of neutral electroweak boson pair production in $13 \mathrm{TeV}$ proton-proton collisions at the LHC, inclusive with respect to any associated hadronic jet activity. The measured integrated and differential cross sections are compared to state-of-the-art theoretical predictions. The agreement is generally good, with some interesting small deviating trends. Some of the measurements are used to search for high-mass new physics effects in an effective anomalous triple gauge coupling approach. No new physics is observed and constraints on the coefficients of the considered anomalous coupling operators are obtained.
\end{abstract}

European Physical Society Conference on High Energy Physics - EPS-HEP2019 -

10-17 July, 2019

Ghent, Belgium

${ }^{*}$ Speaker. 


\section{Introduction and motivation}

ATLAS [1, 2] measurements of neutral diboson production at the LHC [3] probe part of the electroweak sector of the Standard Model (SM), governed by an $\mathrm{SU}(2)_{L} \times \mathrm{U}(1)_{Y}$ gauge symmetry. Apparent deviations from this gauge structure in the measurements could be traced back to physics beyond the Standard Model. This is done by parametrising new physics by effective anomalous triple gauge couplings, or by using an effective field theory where new physics at a higher scale is treated as having no dynamics at the experimentally reached energies, but modifying the effective couplings between dynamical SM fields. The production of dibosons is a particularly important test of the SM electroweak symmetry breaking mechanism, because it is rendered finite at high energy scales by the presence of the Higgs boson.

Throughout this article, lepton $(\ell)$ refers to an electron or a muon, i.e. the types of lepton that can be observed undecayed in ATLAS. We present the following measurements:

- $Z^{(*)} / \gamma^{*} Z^{(*)} / \gamma^{*} \rightarrow \ell^{+} \ell^{-} \ell^{\prime+} \ell^{\prime-}$ [4], across a wide range of the four-lepton invariant mass, which is also the main observable of interest. The presence of the photon, $Z$ boson (produced singly or in pairs), and the Higgs boson is apparent. Several interpretations of the measurement in terms of SM parameters and searches for new physics are performed.

- $Z Z \rightarrow \ell^{+} \ell^{-} v \bar{v}$ [5]: integrated and differential cross sections are presented along with a search for anomalous triple gauge couplings.

- $Z \gamma \rightarrow v \bar{v} \gamma$ [6]: differential cross sections are presented along with a search for anomalous triple gauge couplings.

- $Z \gamma \rightarrow \ell^{+} \ell^{-} \gamma$ [7]: differential cross sections are presented.

Each measurement uses $36 \mathrm{fb}^{-1}$ of proton-proton collision data, except for the $Z \gamma \rightarrow \ell^{+} \ell^{-} \gamma$ measurement, which uses the full ATLAS Run 2 dataset of $139 \mathrm{fb}^{-1}$. The measurements are inclusive with respect to associated jets, as opposed to e.g. dedicated measurements of vector boson scattering, in which the use of jets is crucial to identify the signal. To limit model dependence in the measurements, the cross sections are corrected to fiducial phase spaces defined in terms of stable $^{1}$ particles. The fiducial phase spaces reflect the acceptance of the detector and the analysis requirements applied to reduce background. This way, model-dependent extrapolation outside of the experimentally accessible phase space is largely avoided.

The experimental results are compared to theoretical predictions in NNLO QCD (and sometimes NLO QCD) from Matrix [8, 9] and MCFM [10-13], NLO QCD predictions matched with a parton shower from Powheg [14-18] and Sherpa [19-24] (which merges various final-state parton multiplicities), loop-induced processes in LO QCD from GG2VV [25, 26], NLO QCD corrections to loop-induced processes computed in Refs. [27, 28], and NLO electroweak corrections from the calculation in Refs. [29, 30].

2. $Z^{(*)} / \gamma^{*} Z^{(*)} / \gamma^{*} \rightarrow \ell^{+} \ell^{-} \ell^{\prime+} \ell^{\prime-}$

The four-lepton measurement [4] is very inclusive and therefore exhibits sensitivity to various production mechanisms. These are visible in Figure 1(a), showing the reconstructed event yields as

\footnotetext{
${ }^{1}$ Here, stable means that they have a lifetime long enough to be detected by ATLAS before decaying.
} 
a function of the four-lepton mass along with predictions for the various contributing subprocesses. The high lepton multiplicity means that the background due to misidentified leptons is very small, allowing the use of loose lepton identification $[31,32]$ and a low $p_{\mathrm{T}}>7 \mathrm{GeV}$ requirement. The backgrounds due to other SM processes are also very small. The $Z$ and Higgs-boson peaks are visible around $91 \mathrm{GeV}$ and $125 \mathrm{GeV}$. Above around $180 \mathrm{GeV}$, the production of two on-shell $Z$ bosons contributes, causing a sharp increase and subsequent downward slope of the cross section.

Figure 1(b) shows the differential cross section after background subtraction and unfolding. It is compared to various theoretical predictions, including a fixed-order NNLO QCD prediction. Overall, the agreement is good, except in the region in and around the Higgs boson peak, where NNLO QCD predicts too low a cross section. This is understood, since large higher-order corrections to the loop-induced Higgs boson production are missing here. The different relative contribution of (anti)quark and gluon-initiated subprocesses apparent in Figure 1(a) make it clear that an - albeit model-dependent - measurement of the $g g \rightarrow \ell^{+} \ell^{-} \ell^{\prime+} \ell^{\prime-}$ contribution relative to the prediction, $\mu_{g g}$, is feasible. This is interesting because of the large dependence of this process' predictions on QCD scale variations. The fitted $g g \rightarrow \ell^{+} \ell^{-} \ell^{\prime+} \ell^{\prime-}$ production strength is found to be $\mu_{g g}=1.3 \pm 0.5$ (expected: $1.0 \pm 0.4$ ) with respect to the NLO prediction. With respect to the LO prediction, the fitted value is $\mu_{g g}=2.7 \pm 0.9$. This clearly shows that the data are much more compatible with the NLO prediction.

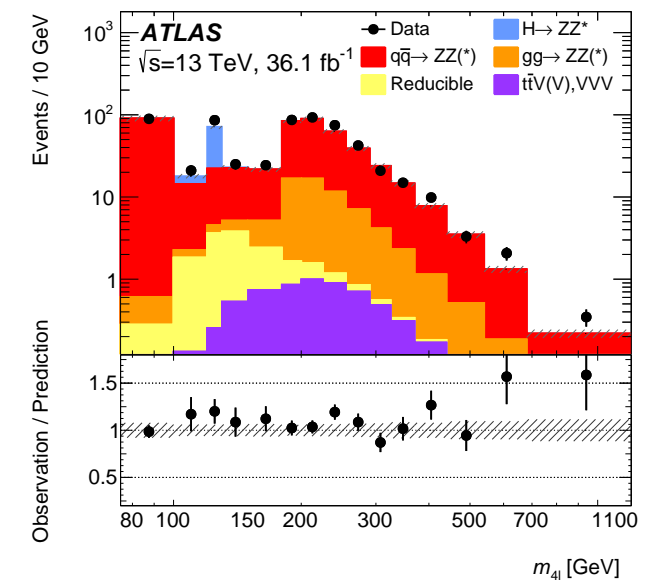

(a)

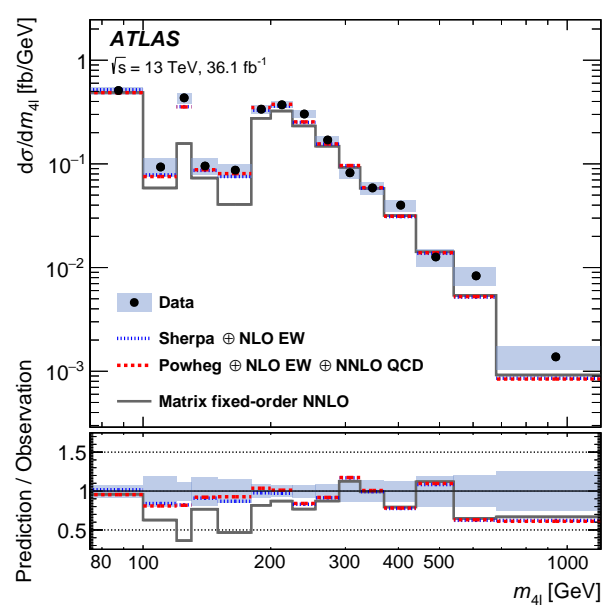

(b)

Figure 1: (a) Reconstructed yields, and (b) differential cross section as a function of the four-lepton invariant mass. Taken from Ref. [4].

Figure 2 shows the double-differential cross section as a function of both the four-lepton mass and a matrix-element discriminant $D_{\mathrm{ME}}$ designed to identify "more ( $s$-channel) Higgs-boson-like" events. These are in the lower panels, while the upper ones show "less ( $s$-channel) Higgs-bosonlike" events. The double-differential cross section in Figure 2 is used to constrain the off-shell Higgs-boson production cross section in the region $m_{4 \ell}>180 \mathrm{GeV}$. An upper limit of 6.5 times the SM prediction is found at the $95 \%$ confidence level. At the same confidence level, the onestandard-deviation range of the expected value is $[4.2,7.2]$. The dedicated ATLAS measurement of off-shell Higgs boson production achieved a somewhat tighter upper limit of 4.5 times the SM 
prediction [33].

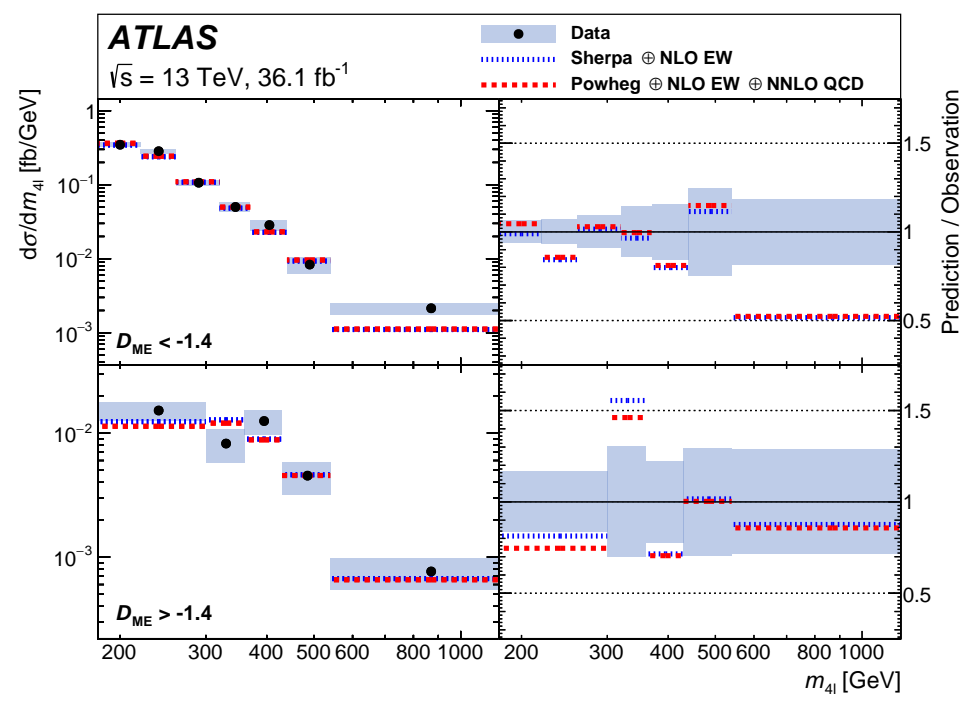

Figure 2: Double-differential cross section as a function of the four-lepton invariant mass and the matrixelement discriminant described in the text. Taken from Ref. [4].

\section{3. $Z Z \rightarrow \ell^{+} \ell^{-} v \bar{v}$}

The measurement of $Z Z$ production in the $\ell^{+} \ell^{-} v \bar{v}$ channel [5] benefits from a branching ratio that is around three times higher than that of the four-lepton channel [4, 34]. Exactly two sameflavour opposite-charge leptons with $p_{\mathrm{T}}>30 \mathrm{GeV}$ and $p_{\mathrm{T}}>20 \mathrm{GeV}$, respectively, passing medium identification are required. The missing transverse energy is required to be $E_{\mathrm{T}}^{\text {miss }}>110 \mathrm{GeV}$. The background is considerably larger than in the four-lepton channel. Around $70 \%$ of the background are from partially identified $W Z \rightarrow \ell^{\prime} v \ell^{+} \ell^{-}$events. The shapes of this background contribution are taken from simulation, while its normalisation is fitted to data in a control region containing three leptons. Background from $Z \rightarrow \ell^{+} \ell^{-}$as well as background in which the dilepton is produced non-resonantly (such as $W W \rightarrow \ell^{+} \ell^{-} v \bar{v}$ ) is greatly reduced by requiring the angular distance of the leptons to be $\Delta R_{\ell^{+} \ell^{-}}<1$.9. Background from $t \bar{t}$ production is suppressed by vetoing events containing a b-tagged jet.

The integrated fiducial cross sections are shown in Table 1 along with predictions. Also shown is the cross section after extrapolation to the entire $Z Z$ phase space with dilepton masses between $66 \mathrm{GeV}$ and $116 \mathrm{GeV}$. This number is in agreement with the extrapolated cross section measured in the $Z Z \rightarrow \ell^{+} \ell^{-} \ell^{\prime+} \ell^{\prime-}$ channel, $\sigma_{Z Z}^{\text {tot }}=17.2 \pm 0.9 \pm 0.6$ (stat) \pm 0.4 (syst) \pm 0.6 (lumi) pb [34]. Differential cross sections as a function of eight different observables are measured. Two examples are shown in Figure 3. The measurements roughly agree with the shown predictions, although there is a hint of some differing trends. The measured cross section with respect to the transverse momentum of the dilepton (Figure 3(b)) is used to search for new physics at high energies using the anomalous triple gauge coupling (aTGC) approach. With no significant deviations from the SM observed, exclusion limits are set on the parameters describing the coupling strength of the effective aTGC vertices. 


\begin{tabular}{cccc}
\hline \hline & & Measured & Predicted \\
\hline \multirow{3}{*}{$\sigma_{Z Z \rightarrow \ell \ell \nu \nu}[\mathrm{fb}]$} & $e e$ & $12.2 \pm 1.0$ (stat) \pm 0.5 (syst) \pm 0.3 (lumi) & $11.2 \pm 0.6$ \\
& $e e+\mu \mu$ & $13.3 \pm 1.0$ (stat) \pm 0.5 (syst) \pm 0.3 (lumi) & $11.2 \pm 0.6$ \\
& $25.4 \pm 1.4$ (stat) \pm 0.9 (syst) \pm 0.5 (lumi) & $22.4 \pm 1.3$ \\
\hline$\sigma_{Z Z}^{\text {tot }}[\mathrm{pb}]$ & Total & $17.8 \pm 1.0$ (stat) \pm 0.7 (syst) \pm 0.4 (lumi) & $15.7 \pm 0.7$ \\
\hline \hline
\end{tabular}

Table 1: Integrated fiducial and extrapolated cross sections along with NNLO QCD predictions with NLO electroweak corrections as well as NLO corrections for the gluon-initiated loop-induced production mode applied. Taken from Ref. [5].

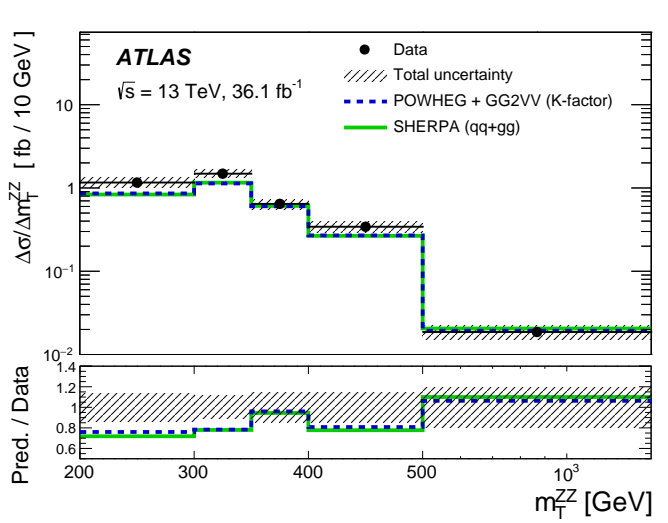

(a)

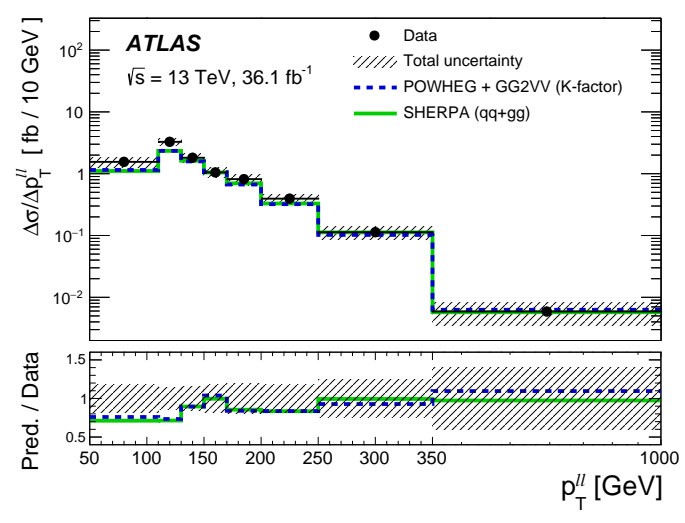

(b)

Figure 3: Differential cross sections with respect to (a) the transverse mass of the $Z Z \rightarrow \ell^{+} \ell^{-} v \bar{v}$ system, and (b) the transverse momentum of the dilepton system. Taken from Ref. [5].

\section{4. $Z \gamma \rightarrow v \bar{v} \gamma$}

For triggering and to suppress background, the $Z \gamma \rightarrow v \bar{v} \gamma$ analysis [6] puts high requirements on the photon transverse energy, $E_{\mathrm{T}}^{\gamma}>150 \mathrm{GeV}$, and the missing transverse energy, $E_{\mathrm{T}}^{\text {miss }}>$ $150 \mathrm{GeV}$. A high $E_{\mathrm{T}}^{\text {miss }}$ significance of $E_{\mathrm{T}}^{\text {miss }} / \sqrt{\sum_{\mathrm{jets}} p_{\mathrm{T}}^{\text {jet }}+E_{\mathrm{T}}^{\gamma}}>10.5 \mathrm{GeV}^{1 / 2}$ helps reduce background with mismeasured $E_{\mathrm{T}}^{\text {miss }}$ and a veto on electrons and muons suppresses backgrounds such as $W+$ jets. Sophisticated data-driven background estimation is used. Background from hadronic jets misidentified as photons is fitted to data in a two-dimensional sideband with inverted photon identification and/or photon isolation. Background with electrons misidentified as photons is obtained by measuring misidentification rates in $Z \rightarrow$ data and applying them to $W \rightarrow v$ events to get the background estimate. Finally, background with a genuine photon and a $W$ boson passing the lepton veto (particularly in the channel $W(\rightarrow \tau v)$ is normalised to a fit in data control regions with one lepton and a small $E_{\mathrm{T}}^{\mathrm{miss}}$ significance, significantly reducing its systematic uncertainty compared to a similar analysis at $8 \mathrm{TeV}[35]$.

Differential fiducial cross sections are measured both without and with jet veto (vetoing jets with $p_{\mathrm{T}}>50 \mathrm{GeV}$ and $\left.|\eta|<4.5\right)$. The latter selection has a higher signal purity, at the expense of signal acceptance. The differential cross section with respect to the photon $E_{\mathrm{T}}$ in both regions is shown in Figure 4 and found to agree with NNLO QCD as well as multijet-merged NLO QCD predictions (the deviation in the highest bin in Figure 4(a) is found to not be statistically significant). 


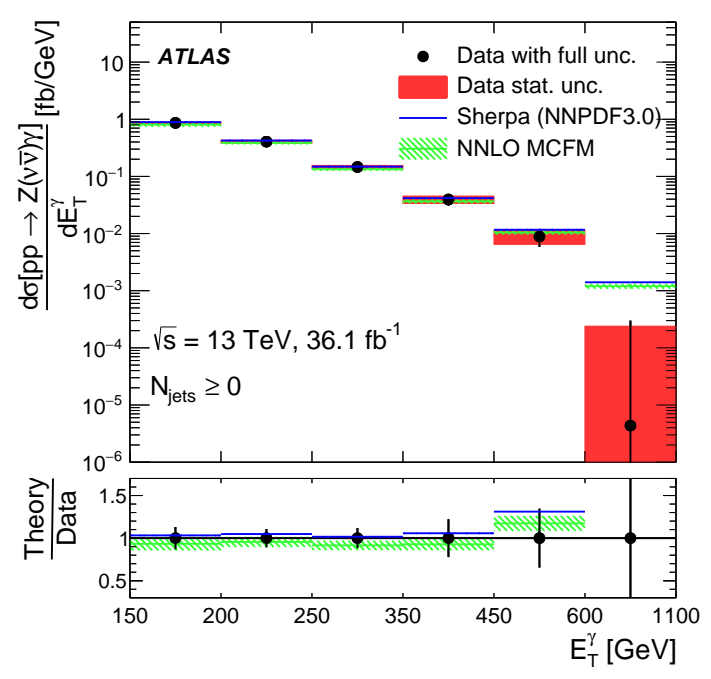

(a)

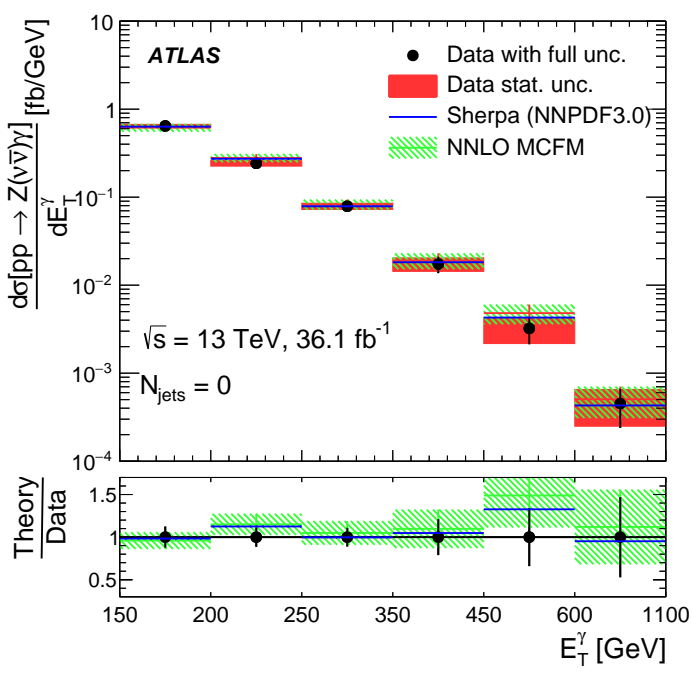

(b)

Figure 4: Transverse energy of the photon in the region (a) without a jet veto, and (b) with a jet veto. Taken from Ref. [6].

The highest bin of the photon $E_{\mathrm{T}}$ distribution in the jet-veto region is used to set the world's tightest exclusion limits on the aTGC parameters governing the $Z Z \gamma$ and $Z \gamma \gamma$ couplings. Only CPconserving couplings are considered, but the limits for the corresponding CP-violating ones are very similar. Examples of limit ellipses are shown in Figure 5.

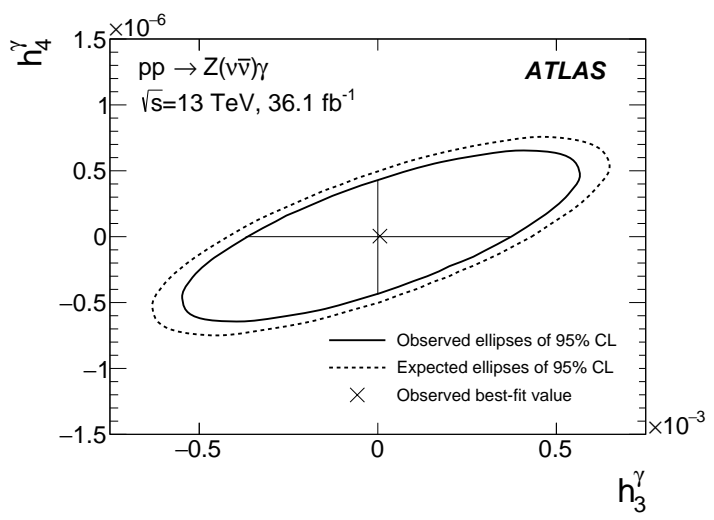

(a)

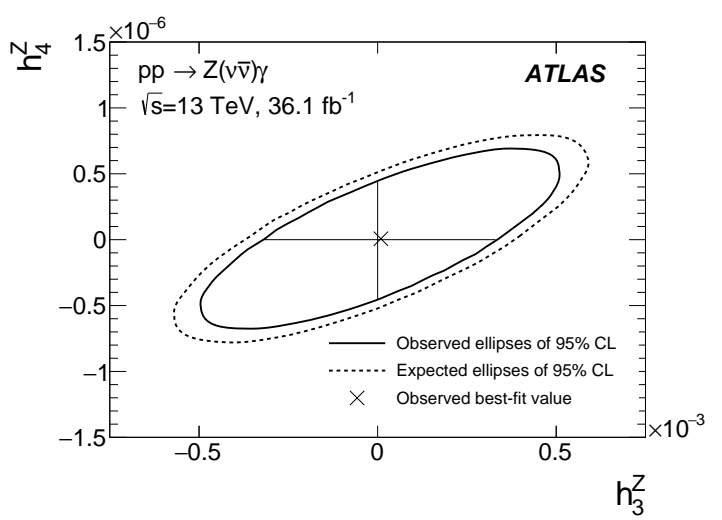

(b)

Figure 5: Exclusion limits on CP-conserving anomalous (a) $Z \gamma \gamma$, and (b) $Z Z \gamma$ coupling parameters. Only the pair of parameters shown in each figure is varied simultaneously, the remaining ones are set to the SM value, i.e. zero. Taken from Ref. [6].

\section{5. $Z \gamma \rightarrow \ell^{+} \ell^{-} \gamma$}

Due to the presence of two leptons, required to have $p_{\mathrm{T}}>30 \mathrm{GeV}$ and $p_{\mathrm{T}}>25 \mathrm{GeV}$, the $Z \gamma \rightarrow \ell^{+} \ell^{-} \gamma$ measurement [7] only requires the photon to have $E_{\mathrm{T}}>30 \mathrm{GeV}-$ five times lower than was necessary in the $Z \gamma \rightarrow v \bar{v} \gamma$ analysis [6]. To ensure that the measurement is dominated by events in which the photon is radiated by an initial-state quark rather than a lepton from the $Z$ 
decay, the mass requirement $m_{\ell \ell}+m_{\ell \ell \gamma}>182 \mathrm{GeV}$ is imposed. The dominant background is due to jets misidentified as photons. This background is determined using a data-driven sideband method and found to be around $10 \%$ of the total expected yield. Pileup background, where the photon and the $Z \rightarrow \ell^{+} \ell^{-}$are produced in independent collisions in the same proton bunch crossing, is estimated by a fit to the $z_{\gamma}-z_{\text {vertex }}$ distribution (where $z$ is the longitudinal coordinate, i.e. along the beam direction). It is found to be around $2 \%$ of the total expected yield.

Differential cross sections as a function of four observables are measured, including the mass of the $\ell \ell \gamma$ system, which is not measurable in the $Z \gamma \rightarrow v \bar{v} \gamma$ analysis. This distribution as well as that of the photon transverse energy is shown in Figure 6. Of the fixed-order predictions shown in the figure, the NNLO QCD calculation agrees substantially better with the data than the NLO QCD calculation, although the difference is mainly in normalisation.

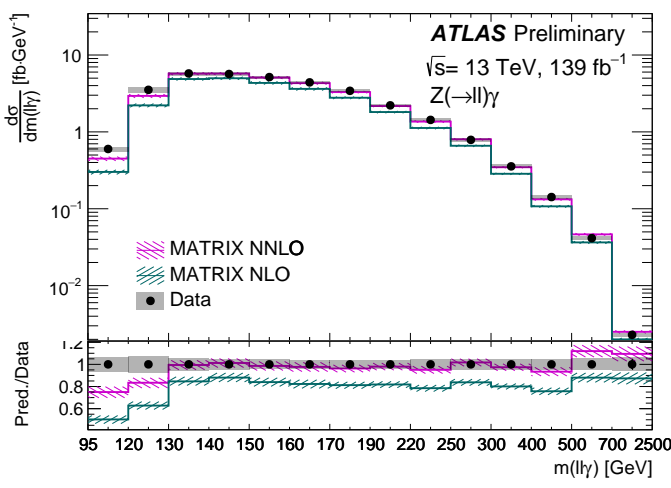

(a)

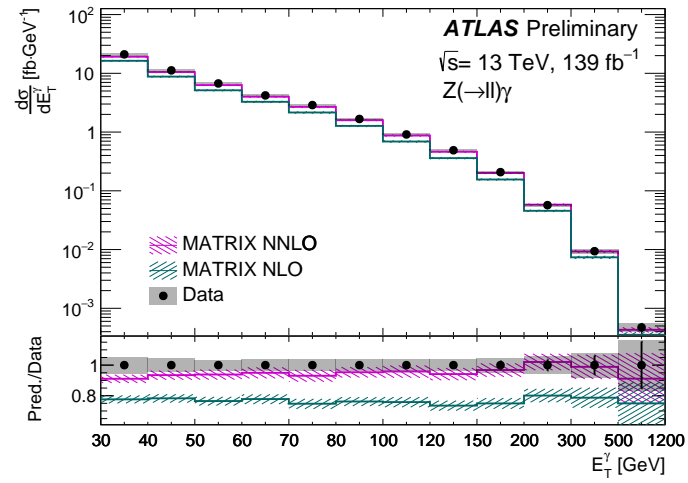

(b)

Figure 6: Differential cross section as a function of (a) the mass of the $\ell \ell \gamma$ system, and (b) the photon transverse energy, compared to fixed-order predictions. Taken from Ref. [7].

\section{Discussion}

Recent ATLAS measurements of inclusive neutral diboson production in $13 \mathrm{TeV}$ proton-proton collisions at the LHC are presented. Several differential cross sections were measured in fiducial regions and show overall agreement with NLO (in some cases jet-multiplicity merged) partonshowered predictions as well as fixed-order NNLO QCD predictions. Various interpretations of the analyses in terms of SM parameters and exclusion limits on neutral aTGCs are performed. The presented analyses highlight the complementarity of studying the $Z \rightarrow v \bar{v}$ and $Z \rightarrow \ell^{+} \ell^{-}$ channels. The former, having a larger branching fraction, are more sensitive to new physics effects at high energy scales (which may be treated in an aTGC effective approach). The latter allow full reconstruction of the final state and looser kinematic requirements, making them excellent for measuring cross sections across a very inclusive phase space. The measurements using $36 \mathrm{fb}^{-1}$ of data are statistically limited, meaning that these measurements will benefit from analysis of the full $139 \mathrm{fb}^{-1}$ collected by ATLAS in LHC Run 2. In the $Z \gamma \rightarrow \ell^{+} \ell^{-} \gamma$ measurement with $139 \mathrm{fb}^{-1}$, the systematic uncertainty dominates over the statistical one in more than half of the bins. The collection of more data as well as future theoretical progress - e.g. the calculation of mixed electroweak and QCD corrections - promise exciting opportunities for new precision tests of the SM with high sensitivity to new physics. 


\section{References}

[1] ATLAS Collaboration, The ATLAS Experiment at the CERN Large Hadron Collider, JINST 3 (2008) S08003.

[2] ATLAS Collaboration, ATLAS Insertable B-Layer Technical Design Report, ATLAS-TDR-19, 2010, URL: https: / / cds . cern.ch/record/1291633, Addendum: ATLAS-TDR-19-ADD-1, 2012, URL: https://cds.cern.ch/record/1451888.

[3] L. Evans and P. Bryant (editors), LHC Machine, JINST 3 (2008) S08001.

[4] ATLAS Collaboration, Measurement of the four-lepton invariant mass spectrum in $13 \mathrm{TeV}$ proton-proton collisions with the ATLAS detector, JHEP 04 (2019) 048, arXiv: 1902.05892 [hep-ex] .

[5] ATLAS Collaboration, Measurement of ZZ production in the levv final state with the ATLAS detector in pp collisions at $\sqrt{s}=13 \mathrm{TeV}$, (2019), arXiv: 1905.07163 [hep-ex] .

[6] ATLAS Collaboration, Measurement of the $Z \gamma \rightarrow v \bar{v} \gamma$ production cross section in pp collisions at $\sqrt{s}=13 \mathrm{TeV}$ with the ATLAS detector and limits on anomalous triple gauge-boson couplings, JHEP 12 (2018) 010, arXiv: 1810.04995 [hep-ex] .

[7] ATLAS Collaboration, Measurement of $Z \gamma \rightarrow \ell^{+} \ell^{-} \gamma$ differential cross-sections in $p p$ collisions at $\sqrt{s}=13 \mathrm{TeV}$ with the ATLAS detector, ATLAS-CONF-2019-034, 2019, URL: https: //cds.cern.ch/record/2682846.

[8] M. Grazzini, S. Kallweit, and M. Wiesemann, Fully differential NNLO computations with MATRIX, Eur. Phys. J. C78 (2018) 537, arXiv: 1711.06631 [hep-ph].

[9] M. Grazzini, S. Kallweit, and D. Rathlev, ZZ production at the LHC: fiducial cross sections and distributions in NNLO QCD, Phys. Lett. B750 (2015) 407, arXiv: 1507.06257 [hep-ph] .

[10] J. M. Campbell and R. K. Ellis, Update on vector boson pair production at hadron colliders, Phys. Rev. D 60 (1999) 113006, arXiv: hep-ph/ 9905386.

[11] J. M. Campbell, R. K. Ellis, and C. Williams, Vector boson pair production at the LHC, JHEP 07 (2011) 018, arXiv: 1105.0020 [hep-ph ] .

[12] J. M. Campbell, R. K. Ellis, and W. T. Giele, A Multi-Threaded Version of MCFM, Eur. Phys. J. C75 (2015) 246, arXiv: 1503.06182 [physics . comp-ph] .

[13] R. Boughezal, J. M. Campbell, R. K. Ellis, C. Focke, W. Giele, X. Liu, F. Petriello, and C. Williams, Color singlet production at NNLO in MCFM, Eur. Phys. J. C77 (2017) 7, arXiv: 1605.08011 [hep-ph]. 
[14] S. Alioli, P. Nason, C. Oleari, and E. Re, A general framework for implementing NLO calculations in shower Monte Carlo programs: the POWHEG BOX, JHEP 06 (2010) 043, arXiv: 1002.2581 [hep-ph] .

[15] S. Frixione, P. Nason, and C. Oleari,

Matching NLO QCD computations with parton shower simulations: the POWHEG method, JHEP 11 (2007) 070, arXiv: 0709.2092 [hep-ph ] .

[16] P. Nason, A new method for combining NLO QCD with shower Monte Carlo algorithms, JHEP 11 (2004) 040, arXiv: hep-ph / 0409146.

[17] T. Melia, P. Nason, R. Rontsch, and G. Zanderighi, $W+W-, W Z$ and ZZ production in the POWHEG BOX, JHEP 11 (2011) 078, arXiv: 1107.5051 [hep-ph].

[18] P. Nason and G. Zanderighi, $W^{+} W^{-}, W Z$ and ZZ production in the POWHEG-BOX-V2, Eur. Phys. J. C74 (2014) 2702, arXiv: 1311.1365 [hep-ph] .

[19] T. Gleisberg, S. Höche, F. Krauss, M. Schönherr, S. Schumann, et al., Event generation with SHERPA 1.1, JHEP 02 (2009) 007, arXiv: 0811.4622 [hep-ph].

[20] T. Gleisberg and S. Höche, Comix, a new matrix element generator, JHEP 12 (2008) 039, arXiv: 0808.3674 [hep-ph].

[21] F. Cascioli, P. Maierhofer, and S. Pozzorini, Scattering Amplitudes with Open Loops, Phys. Rev. Lett. 108 (2012) 111601, arXiv: 1111.5206 [hep-ph] .

[22] S. Catani, F. Krauss, R. Kuhn, and B. R. Webber, QCD matrix elements + parton showers, JHEP 11 (2001) 063, arXiv: hep-ph/ 0109231.

[23] S. Höche, F. Krauss, M. Schönherr, and F. Siegert, QCD matrix elements + parton showers: The NLO case, JHEP 04 (2013) 027, arXiv: 1207.5030 [hep-ph].

[24] S. Schumann and F. Krauss,

A parton shower algorithm based on Catani-Seymour dipole factorisation, JHEP 03 (2008) 038, arXiv: 0709.1027 [hep-ph ] .

[25] N. Kauer and G. Passarino,

Inadequacy of zero-width approximation for a light Higgs boson signal, JHEP 08 (2012) 116, arXiv: 1206.4803 [ hep-ph ] .

[26] N. Kauer,

Interference effects for $H \rightarrow W W / Z Z \rightarrow \ell \bar{v}_{\ell} \bar{\ell} v_{\ell}$ searches in gluon fusion at the $L H C$, JHEP 12 (2013) 082, arXiv: 1310 . 7011 [hep-ph] .

[27] F. Caola, K. Melnikov, R. Röntsch, and L. Tancredi, $Q C D$ corrections to ZZ production in gluon fusion at the $L H C$, Phys. Rev. D92 (2015) 094028, arXiv: 1509.06734 [hep-ph] . 
[28] F. Caola, M. Dowling, K. Melnikov, R. Röntsch, and L. Tancredi, QCD corrections to vector boson pair production in gluon fusion including interference effects with off-shell Higgs at the LHC, JHEP 07 (2016) 087, arXiv: 1605.04610 [hep-ph].

[29] B. Biedermann, A. Denner, S. Dittmaier, L. Hofer, and B. Jäger, Electroweak corrections to $p p \rightarrow \mu^{+} \mu^{-} e^{+} e^{-}+X$ at the LHC: a Higgs background study, Phys. Rev. Lett. 116 (2016) 161803, arXiv: 1601.07787 [hep-ph] .

[30] B. Biedermann, A. Denner, S. Dittmaier, L. Hofer, and B. Jäger, Next-to-leading-order electroweak corrections to the production of four charged leptons at the LHC, JHEP 01 (2017) 033, arXiv: 1611.05338 [ hep-ph ] .

[31] ATLAS Collaboration, Electron reconstruction and identification in the ATLAS experiment using the 2015 and 2016 LHC proton-proton collision data at $\sqrt{s}=13 \mathrm{TeV}$, Eur. Phys. J. (2019), arXiv: 1902.04655 [hep-ex] .

[32] ATLAS Collaboration, Muon reconstruction performance of the ATLAS detector in proton-proton collision data at $\sqrt{s}=13$ TeV, Eur. Phys. J. C 76 (2016) 292, arXiv: 1603.05598 [hep-ex] .

[33] ATLAS Collaboration, Constraints on off-shell Higgs boson production and the Higgs boson total width in $Z Z \rightarrow 4 \ell$ and $Z Z \rightarrow 2 \ell 2 v$ final states with the ATLAS detector, Phys. Lett. B 786 (2018) 223, arXiv: 1808.01191 [hep-ex] .

[34] ATLAS Collaboration, $Z Z \rightarrow \ell^{+} \ell^{-} \ell^{\prime+} \ell^{--}$cross-section measurements and search for anomalous triple gauge couplings in $13 \mathrm{TeV}$ pp collisions with the ATLAS detector, Phys. Rev. D 97 (2018) 032005, arXiv: 1709.07703 [hep-ex] .

[35] ATLAS Collaboration, Measurements of $Z \gamma$ and $Z \gamma \gamma$ production in pp collisions at $\sqrt{s}=8$ TeV with the ATLAS detector, Phys. Rev. D 93 (2016) 112002, arXiv: 1604.05232 [hep-ex] .

[36] ATLAS Collaboration, ATLAS-TDR-19-ADD-1, 2012, URL: https://cds.cern.ch/record/1451888. 